\title{
Orthostatic hypertension as a predisposing factor for masked hypertension: the J-SHIPP study
}

\author{
Yasuharu Tabara ${ }^{1,2}$, Michiya Igase ${ }^{2}$, Tetsuro Miki ${ }^{1,2}$, Yasumasa Ohyagi ${ }^{2}$, Fumihiko Matsuda ${ }^{1}$, \\ Katsuhiko Kohara ${ }^{2,3}$ and the J-SHIPP study group ${ }^{4}$
}

\begin{abstract}
Masked hypertension (HT) is a known risk factor for cardiovascular outcomes. Postural blood pressure (BP) dysregulation is another BP phenomenon representing cardiovascular frailty. Given their several shared risk factors, we suspected an interrelationship between these two BP phenomena. Here we investigated a possible relationship between masked HT and postural BP dysregulation in a general population. Study subjects were 884 apparently healthy individuals (aged $66.3 \pm 8.9$ years). Masked HT was assessed on the basis of the ambulatory monitored average awake BP and office-measured BP values. Orthostatic BP change was measured at our office after a subject was asked to actively stand up. A strong inverse relationship was noted for orthostatic systolic BP (SBP) change and office-to-awake SBP differences (office-awake BP) $(r=-0.422$, $P<0.001)$, and these relationships were replicated in the second-visit measurements $(n=101, r=-0.326, P=0.001)$. Multivariate analysis revealed that the inverse association was independent $(\beta=-0.23, P<0.001)$ of possible covariates, including baseline office BP and antihypertensive treatment. Orthostatic HT (OHT), which is defined as postural increases in SBP $>10 \mathrm{~mm} \mathrm{Hg}, 3 \mathrm{~min}$ after standing $(P=0.001)$, but not transient $\mathrm{HT}$ at only $1 \mathrm{~min}(P=0.767)$, was associated with greater office-to-awake SBP differences than in orthostatic normotensive subjects. Among apparently normotensive subjects, the frequency of masked HT was therefore significantly greater in subjects who showed OHT 3 min after standing (52.1\%) compared with controls $(27.5 \%$ ) (odds ratio $=3.01, P=0.001$ ). We observed an intra-individual relationship between the postural $\mathrm{BP}$ change and the office-to-awake BP differences, and subjects who showed OHT were likely to have masked HT irrespective of antihypertensive treatment. Hypertension Research (2016) 39, 664-669; doi:10.1038/hr.2016.43; published online 16 June 2016
\end{abstract}

Keywords: masked hypertension; orthostatic blood pressure change; orthostatic hypertension

\section{INTRODUCTION}

Masked hypertension (HT) is a combined phenomenon of normal to high-normal office-measured blood pressure (BP) with a homemeasured BP exceeding a cutoff maximum for out-of-office-measured daytime BP (that is, systolic BP (SBP) > $135 \mathrm{~mm} \mathrm{Hg}$ or diastolic BP (DBP) $>85 \mathrm{~mm} \mathrm{Hg}){ }^{1}$ A meta-analysis from the International DAtabase on ambulatory BP in relation to Cardiovascular Outcomes $(\text { IDACO })^{2}$ reported that masked HT might be an independent risk factor for the incidence of cardiovascular diseases irrespective of antihypertensive treatment status. Further, masked HT has been suggested to be associated with subclinical organ damage, namely, left ventricular hypertrophy ${ }^{3}$ and atherosclerosis measured at the carotid artery. ${ }^{4}$ Although the frequency of masked HT varies by population, an IDACO study in a general population of 9691 subjects $^{5}$ has reported a frequency of $18.8 \%$ in untreated non-diabetic subjects and $30.5 \%$ in antihypertensive-treated subjects. High-normal BP, older age, obesity and diabetes ${ }^{5,6}$ have been suggested as determinants for masked HT. However, the estimated contribution ratio of these factors is $<20 \%,{ }^{6}$ thus necessitating further studies to clarify the clinical background of masked HT.

Orthostatic BP dysregulation is another BP parameter known to represent cardiovascular frailty. Orthostatic hypotension (OHYPO) is a common phenomenon, particularly among the elderly, and has been suggested to be associated with the incidence of stroke, ${ }^{7}$ cardiovascular events $^{8,9}$ and mortality. ${ }^{10,11}$ Furthermore, previous epidemiological studies, ${ }^{12,13}$ including one of our own, ${ }^{14}$ have identified not only OHYPO but also orthostatic HT (OHT) as potential cardiovascular risks. A major cause of postural BP dysregulation is atherosclerosis and the consequent insufficient baroreceptor-mediated compensatory reaction, namely, increasing heart rate, myocardial contractility and peripheral vasoconstriction. ${ }^{15}$ An older age, HT, peripheral vascular disease, smoking and an unfavorable metabolic profile have also been suggested as factors that might disrupt postural BP regulation. ${ }^{16}$ Given that several of these risk factors are common to both postural BP

${ }^{1}$ Center for Genomic Medicine, Kyoto University Graduate School of Medicine, Kyoto, Japan; ${ }^{2}$ Department of Geriatric Medicine, Ehime University Graduate School of Medicine, Ehime, Japan and ${ }^{3}$ Department of Neurology, National Hospital Organization Ehime Medical Center, Ehime, Japan

${ }^{4}$ Members of the J-SHIPP study group are listed before references.

Correspondence: Dr Y Tabara, Center for Genomic Medicine, Kyoto University Graduate School of Medicine, Shogoinkawara-cho 53, Sakyo-ku, Kyoto 606-8507, Japan.

E-mail: tabara@genome.med.kyoto-u.ac.jp

Received 25 January 2016; revised 10 March 2016; accepted 17 March 2016; published online 16 June 2016 
dysregulation and masked HT, we hypothesized an inter-relationship between the two phenomena. However, whereas a small-scale case-control study has suggested a possible association between OHT and masked $\mathrm{HT},{ }^{17}$ no data from a larger general population have yet been obtained.

Identification of masked $\mathrm{HT}$ requires $\mathrm{BP}$ monitoring at home or ambulatory BP monitoring (ABPM). Understanding which clinical characteristics potentiality indicate masked HT is therefore useful in identifying subjects in need of monitoring BP levels and subjects at risk for cardiovascular frailty.

Here, to clarify the possible inter-relationship between orthostatic BP dysregulation and masked HT, we conducted a cross-sectional study analyzing a data set from the Shimanami Health Promoting Program (J-SHIPP study), which is a longitudinal study consisting of several population-based cohorts.

\section{METHODS}

\section{Study subjects}

The J-SHIPP study was conducted in Ehime Prefecture, in the eastern part of Japan, and includes several general population cohorts. We analyzed a data set of apparently healthy middle-aged to elderly consecutive participants in the medical check-up program at Ehime University Hospital Anti-aging Center from February 2006 to November 2014. This medical check-up is provided to general residents of Ehime Prefecture and is specifically designed to evaluate aging-related disorders, including atherosclerosis, cardiovascular disease, physical function and cognitive function. ${ }^{18,19}$ Recruitment was performed via mass communications, such as internet homepages, periodical newspapers in the local community and commercial newspapers, and a total of 1897 physically independent individuals gave written informed consent to participate in this study. From these willing participants, individuals who did not undergo ABPM $(n=1001)$, who were undergoing insulin therapy $(n=2)$ and whose clinical parameters used in this study were not fully available $(n=10)$ were excluded from this study. ABPM was provided as an optional examination upon request. All study procedures were approved by the ethics committee of the Ehime University Graduate School of Medicine.

\section{Repeated measurement}

Second-visit measurements in individuals who participated in the medical check-up program multiple times of their own volition and underwent ABPM measurements $(n=101)$ were used in the replication analysis. The mean ( \pm s.d.) duration between the first and second visit was $857 \pm 546$ days.

\section{Measurement of office BP and orthostatic BP change}

Basal office BP was measured by using an automatic cuff-oscillometric device (HEM-9000AI; Omron Healthcare, Kyoto, Japan) after subjects had spent at least $5 \mathrm{~min}$ resting in a sitting position. Subjects were then asked to stand up without any assistance, and measurements of orthostatic BP were performed at 1 and 3 min after postural change.

The HEM-9000AI can measure brachial BP simultaneously via the cuffoscillometric method and via the radial arterial waveform using a tonometric sensor to estimate central BP. In the present study, measurements on subjects in a sitting position were taken for both brachial BP and radial arterial waveforms, and the brachial BP alone was then measured with subjects in a standing position after detachment of the tonometric sensor.

OHYPO and OHT were defined as a maximum postural SBP change of $\leqslant-20 \mathrm{~mm} \mathrm{Hg}$ and $\geqslant 10 \mathrm{~mm} \mathrm{Hg}$ at either standing measurement, respectively (Supplementary Figure S1).

\section{4-h ambulatory BP monitoring}

ABPM was taken within 1 month after the medical check-up ( $5.1 \pm 5.9$ days) by using a cuff-oscillometric device (TM-2431; A\&D, Tokyo, Japan), which is a fully automatic device that was preset to measure BP and heart rate every 15 min during the day (0700-2000 hours) and every $30 \mathrm{~min}$ at night. Outlier ambulatory BP readings were rejected by using an A\&D method, which is a commonly used simple rejection method that excludes BP readings outside of a specified range. ${ }^{20}$ Sleep duration was assessed by individual interview, and overall averages of $24-\mathrm{h}$, awake and sleeping ambulatory BP values were calculated for each individual.

\section{Definition of masked, white-coat and sustained HT}

Masked, white-coat and sustained HT were defined on the basis of office $\mathrm{BP}$ and average awake BP. Office HT was defined as clinic-measured $\mathrm{SBP} \geqslant 140 \mathrm{~mm} \mathrm{Hg}$ or $\mathrm{DBP} \geqslant 90 \mathrm{~mm} \mathrm{Hg}$, whereas awake HT was defined as an average awake $\mathrm{SBP} \geqslant 135 \mathrm{~mm} \mathrm{Hg}$ or $\mathrm{DBP} \geqslant 85 \mathrm{~mm} \mathrm{Hg}$, irrespective of antihypertensive treatment status. ${ }^{1}$ Masked, white-coat and sustained HT were then, respectively, defined as awake HT and office NT, awake NT and office HT and awake HT and office HT.

\section{Clinical parameters}

Other clinical parameters used in this study were obtained from a personal record evaluated through the medical check-up program. Types of antihypertensive drugs regularly used by subjects were investigated by consulting a personal booklet for medication, which is generally provided to patients by pharmacies to explain effectiveness and to encourage the safe use of medications. The history of cardiovascular diseases, including symptomatic stroke, myocardial infarction and angina pectoris, was assessed using a structured questionnaire.

\section{Statistical analysis}

Differences in numerical variables were assessed by using an analysis of variance, and subsequent post-hoc analysis was performed via Dunnett's test. Frequency differences were assessed with the chi-squared test, and the concordance of subgroups was evaluated on the basis of the $\kappa$ coefficient. Factors independently associated with orthostatic SBP change and office-tohome SBP differences were identified via linear regression analysis. All statistical analyses were conducted using the commercially available statistical software (JMP version 9.0.2; SAS Institute, Cary, NC, USA), with $P$-values $<0.05$ considered significant.

\section{RESULTS}

Subjects' clinical characteristics, including orthostatic BP changes, are shown in Table 1. Frequencies of masked, white-coat and sustained HT were 17.0, 15.2 and 28.1\%, respectively (Supplementary Table S1). Distributions of maximum orthostatic SBP change are shown in Supplementary Figure S1. With respect to postural change, mean SBP was slightly deceased, whereas heart rate was slightly increased (Table 1). Mean awake SBP was slightly lower than that measured at the office. Despite excluding a large number of subjects because of unavailability of ABPM data, we noted no marked differences in clinical parameters between the included and excluded subjects except that the included study subjects had a slightly higher mean SBP than excluded subjects (Supplementary Table S2).

Relationships among clinical parameters, orthostatic changes in SBP and office-to-awake SBP differences are summarized in Supplementary Tables S3 and S4. Factors showing the most marked association with orthostatic SBP change were sex, body mass index, basal BPs measured at the office and homeostasis model assessment index, whereas no clear relationships were observed with other glycemic or lipid parameters. In contrast, age was significantly associated with only office-to-awake SBP differences, except for office-measured BP levels and awake BP levels. Subjects taking antihypertensive medication had slightly greater office-to-awake SBP differences but not orthostatic SBP change compared with subjects not taking medication (Supplementary Table S5).

Figure la shows the intra-individual relationships between orthostatic SBP change and office-to-awake SBP differences. A strong inverse association was observed between these two parameters, and 
Table 1 Clinical characteristics of study subjects $(n=884)$

\begin{tabular}{|c|c|c|c|}
\hline Age (years) & & \multicolumn{2}{|l|}{$66.3 \pm 8.9$} \\
\hline Sex (male/female) & & \multicolumn{2}{|l|}{$362 / 522$} \\
\hline BMI $\left(\mathrm{kg} \mathrm{m}^{-2}\right)$ & & \multicolumn{2}{|l|}{$23.2 \pm 3.0$} \\
\hline History of CVD (\%) & & \multicolumn{2}{|l|}{4.8} \\
\hline \multirow[t]{2}{*}{ Office $B P$} & & \multicolumn{2}{|c|}{ Orthostatic BP } \\
\hline & & $1 \mathrm{~min}$ & $3 \mathrm{~min}$ \\
\hline Systolic (mm Hg) & $136 \pm 20$ & $133 \pm 19$ & $132 \pm 19$ \\
\hline Diastolic (mm Hg) & $77 \pm 12$ & $80 \pm 11$ & $79 \pm 11$ \\
\hline HR (beats per min) & $66 \pm 10$ & $71 \pm 11$ & $71 \pm 11$ \\
\hline Ambulatory $B P$ & Awake & \multicolumn{2}{|c|}{ Sleeping } \\
\hline Systolic (mm Hg) & $133 \pm 14$ & \multicolumn{2}{|c|}{$117 \pm 16$} \\
\hline Diastolic (mm Hg) & $79 \pm 8$ & \multicolumn{2}{|c|}{$69 \pm 9$} \\
\hline HR (beats per min) & $72 \pm 8$ & \multicolumn{2}{|c|}{$60 \pm 8$} \\
\hline \multicolumn{4}{|l|}{ Antihypertensive medications } \\
\hline Diuretics (\%) & \multicolumn{3}{|c|}{3.1} \\
\hline Alpha-blockers (\%) & \multicolumn{3}{|c|}{1.4} \\
\hline Beta-blockers (\%) & \multicolumn{3}{|c|}{4.3} \\
\hline Calcium channel blockers (\%) & \multicolumn{3}{|c|}{24.1} \\
\hline ACE inhibitors (\%) & \multicolumn{3}{|c|}{1.7} \\
\hline Angiotensin-receptor blockers (\%) & \multicolumn{3}{|c|}{18.9} \\
\hline Nitrates $(\%)$ & \multicolumn{3}{|c|}{0.3} \\
\hline Any classes (\%) & \multicolumn{3}{|c|}{36.2} \\
\hline \multicolumn{4}{|l|}{ Glycemic factors } \\
\hline Glucose $\left(\mathrm{mg} \mathrm{dl}^{-1}\right)$ & \multicolumn{3}{|c|}{$102 \pm 16$} \\
\hline Insulin $\left(\mu \cup \mathrm{ml}^{-1}\right)$ & \multicolumn{3}{|c|}{$5.8 \pm 3.5$} \\
\hline HOMA-IR & \multicolumn{3}{|c|}{$1.50 \pm 1.03$} \\
\hline \multicolumn{4}{|l|}{ Plasma lipids } \\
\hline Triglyceride $\left(\mathrm{mg} \mathrm{dl}^{-1}\right)$ & \multicolumn{3}{|c|}{$107 \pm 59$} \\
\hline Total cholesterol ( $\left.\mathrm{mg} \mathrm{dl}^{-1}\right)$ & \multicolumn{3}{|c|}{$214 \pm 36$} \\
\hline HDL cholesterol $\left(\mathrm{mg} \mathrm{dl}^{-1}\right)$ & \multicolumn{3}{|c|}{$66 \pm 17$} \\
\hline
\end{tabular}

Abbreviations: ACE, angiotensin-converting enzyme; BMI, body mass index; BP, blood pressure; CVD, cardiovascular disease; HDL, high-density lipoprotein; HOMA-IR, homeostasis model assessment of insulin resistance; HR, heart rate.

Values are mean \pm s.d.

CVD includes symptomatic stroke, myocardial infarction and angina pectoris. HOMA-IR was calculated by the following equation: (glucose $x$ insulin)/405.

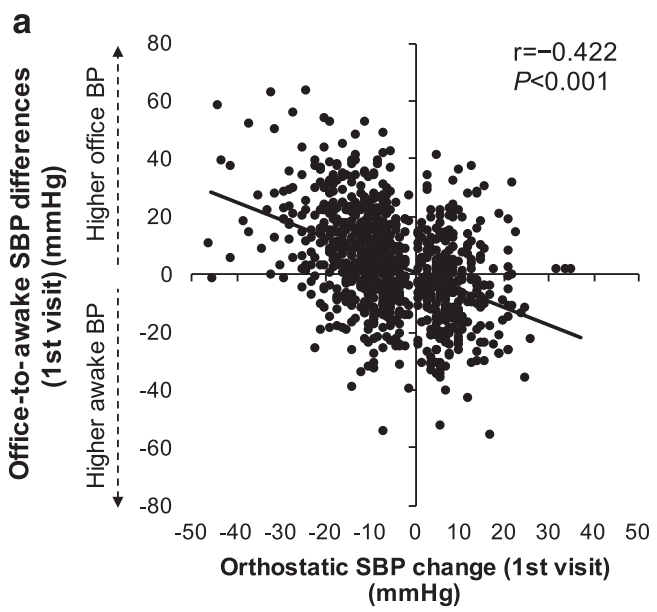

increases in orthostatic SBP were associated with higher awake SBP, whereas decreases were associated with higher office SBP. These relationships were validated at the second-visit measurement in a small population of subjects (Figure 1b) with a slightly lower mean BP than that of all subjects measured at the first visit (Supplementary Table S6), despite poor reproducibility of the orthostatic SBP change in the categorical (Figure 2a) and numerical analyses (Supplementary Figure S2A). In contrast, office-to-awake BP differences had moderate reproducibility (Figure 2b, Supplementary Figure S2B).

To further clarify the relationship between postural SBP change and office-to-awake SBP differences, a multiple linear regression analysis was performed (Table 2). The results indicated that the inverse association between orthostatic changes in SBP and office-to-awake SBP differences was independent (Model 1 in Table 2) of possible covariates, including basal office SBP, and the association was stronger when orthostatic SBP change was included in the regression model as an independent variable (Model 2 in Table 2). This association remained significant in analyses subdivided by antihypertensive medication status (treated subjects: $\beta=-0.28, P<0.001$; untreated subjects: $\beta=-0.19, P<0.001)$.

The effects of the timing of orthostatic BP measurement on office-to-awake SBP differences are shown in Figure 3. OHT or OHYPO $3 \mathrm{~min}$ after standing was associated with greater office-to-awake SBP differences, whereas transient increases or decreases in SBP only at 1 min after standing did not show any significant association with office-to-home SBP differences.

To clarify the diagnostic significance of OHT in ostensibly normotensive subjects, namely, normotensive $(n=352)$ and masked hypertensive $(n=150)$ subjects, the frequency differences of masked HT predicted by orthostatic SBP status are shown in Figure 4. The frequency of masked HT was significantly greater in subjects who showed OHT $3 \mathrm{~min}$ after standing compared with the controls. Although the masked hypertensive subjects, compared with the controls, tended to be slightly older and male (Supplementary Table S7), the greater frequency of masked HT in the OHT subgroup compared with the controls was statistically significant, even after adjustment for covariates such as age, sex, body mass index and basal SBP (Figure 4). This association remained significant even after further adjustment for antihypertensive medication $(P=0.001)$ and in

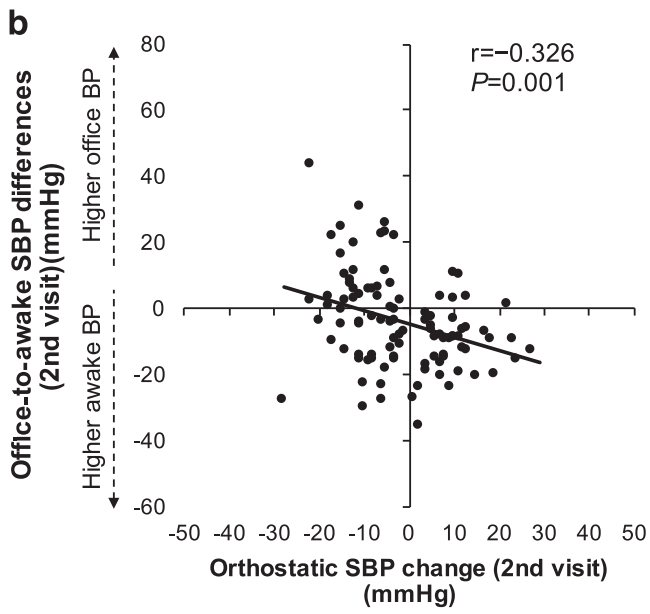

Figure 1 Scatter plots of orthostatic SBP change vs. the office-to-awake SBP difference. Orthostatic BP was measured 1 and 3 min after standing, and the maximum change from the baseline seated BP was considered the orthostatic BP change. Office-to-awake BP differences were calculated by subtracting the average awake BP from office-measured BP (a) at first visit $(n=884)$ and (b) at second visit $(n=101)$. 
a

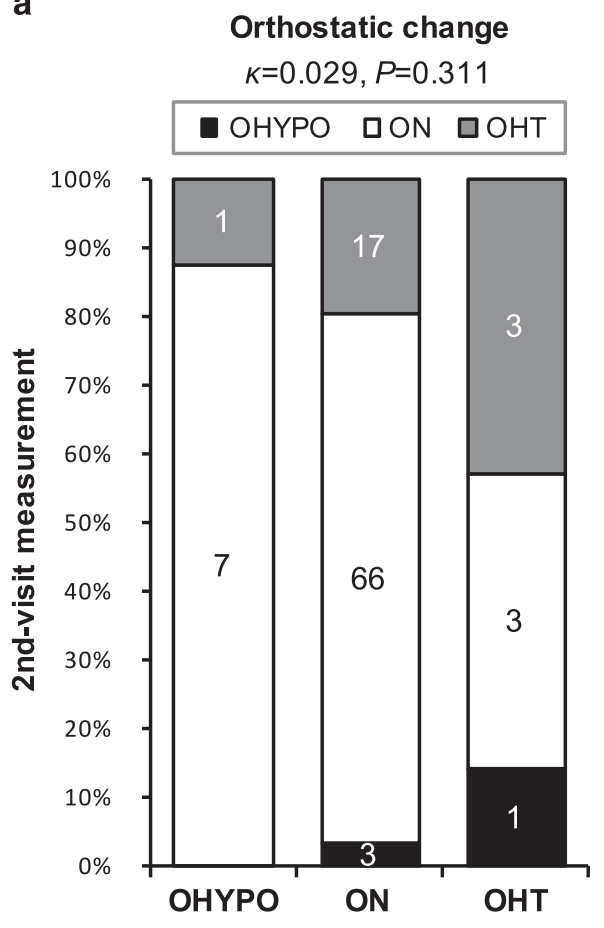

b

Masked, white-coat, sustained HT $\kappa=0.273, P<0.001$

$\square$ NT $\square$ Masked HT $\square$ WCHT $\quad \mathbf{H T}$

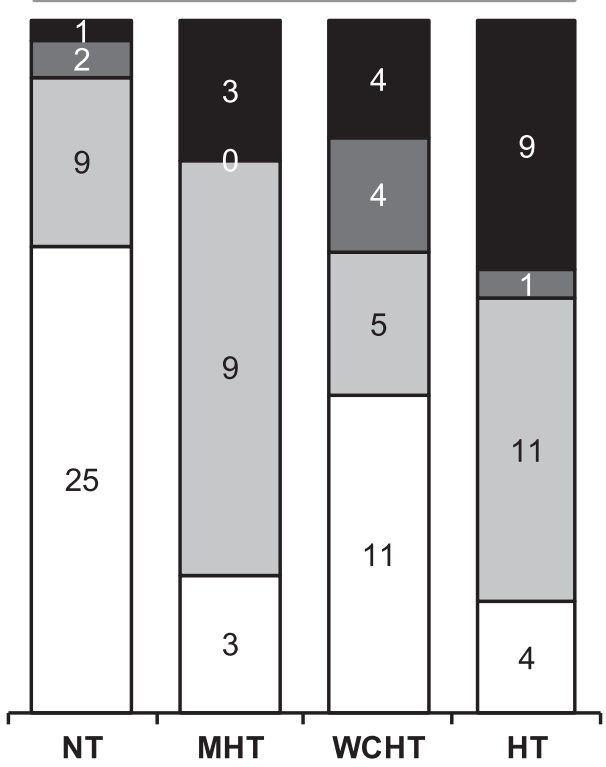

1st-visit measurement

Figure 2 Intra-individual reproducibility of BP pattern $(n=101)$. (a) Orthostatic hypertension (OHT) and hypotension (OHYPO) were defined as a maximum postural SBP change $\geqslant 10 \mathrm{~mm} \mathrm{Hg}$ and $\leqslant-20 \mathrm{~mm} \mathrm{Hg}$, respectively. (b) Sustained HT (HT), office HT and awake HT; white-coat HT (WCHT), office HT and awake normotension (NT); masked HT (MHT), office NT and awake HT; NT, office NT and awake NT. Office HT was defined as SBP $\geqslant 140 \mathrm{~mm}$ Hg or $\mathrm{DBP} \geqslant 90 \mathrm{~mm} \mathrm{Hg}$. Awake HT was defined as SBP $\geqslant 135 \mathrm{~mm} \mathrm{Hg}$ or DBP $\geqslant 85 \mathrm{~mm} \mathrm{Hg}$. Numbers of subjects in each subgroup are shown in the columns. Concordance of the categories in both periods was evaluated by using a $\kappa$ coefficient.

Table 2 Multiple linear regression analysis for SBP alterations

\begin{tabular}{|c|c|c|c|c|}
\hline & \multicolumn{2}{|c|}{ Model 1} & \multicolumn{2}{|c|}{ Model 2} \\
\hline & \multicolumn{2}{|c|}{$\begin{array}{l}\text { Orthostatic SBP } \\
\text { change }(\mathrm{mm} \mathrm{Hg})\end{array}$} & \multicolumn{2}{|c|}{$\begin{array}{l}\text { Office-to-awake SBP } \\
\text { difference }(\mathrm{mm} \mathrm{Hg})\end{array}$} \\
\hline & $\beta$ & P-value & $\beta$ & P-value \\
\hline Age (years) & 0.04 & 0.407 & -0.05 & 0.268 \\
\hline Sex (male) & 1.94 & $<0.001$ & -0.52 & 0.226 \\
\hline BMI $\left(\mathrm{kg} \mathrm{m}^{-2}\right)$ & 0.40 & 0.002 & -0.55 & $<0.001$ \\
\hline Antihypertensive medication & 0.65 & 0.102 & -0.22 & 0.615 \\
\hline Baseline office SBP (mm Hg) & -0.12 & $<0.001$ & 0.61 & $<0.001$ \\
\hline Orthostatic SBP change (mm Hg) & & & -0.23 & $<0.001$ \\
\hline Office-to-awake BP difference (mm Hg) & \multicolumn{2}{|c|}{$-0.19<0.001$} & \multirow{2}{*}{\multicolumn{2}{|c|}{561}} \\
\hline$R^{2}$ & \multicolumn{2}{|c|}{0.230} & & \\
\hline
\end{tabular}

Abbreviations: BP, bood pressure; SBP, systolic BP.

$\beta$ and $R^{2}$ represent the unstandardized regression coefficient and the coefficient of determination, respectively.

analyses subdivided by antihypertensive treatment (treated: $P=0.003$; untreated: $P=0.042$ ).

\section{DISCUSSION}

In this cross-sectional study in a general population, we clarified a significant intra-individual relationship between orthostatic BP change and office-to-awake BP differences. In apparently normotensive subjects, the frequency of masked HT was significantly greater in subjects who showed OHT 3 min after standing.
OHYPO has been suggested to be a prognostic marker of cardiovascular outcomes and mortality, particularly in frail elderly individuals. ${ }^{7-11}$ Orthostatic BP dysregulation (both OHYPO and OHT) is believed to represent the attenuation of BP homeostasis control and to be a risk marker for cardiovascular outcomes. ${ }^{12,13}$ Although the suggested mechanisms underlying OHT (for example, excessive compensatory reaction to orthostatic stress) differ slightly from those of OHYPO, the reported common pathophysiological features between OHYPO and OHT-namely, atherosclerotic vascular change-suggest prognostic similarity between these BP phenomena. However, in the present study, we showed that subjects with OHT were likely to have a higher awake BP than OHYPO and orthostatic normotensive subjects, independently of office-measured BP levels and antihypertensive treatment. Because masked HT is a strong risk factor for cardiovascular outcomes - on par with sustained $\mathrm{HT}^{2}$ - masked HT might be involved to some degree in the epidemiological relationship between OHT and cardiovascular outcomes. OHT should therefore be considered to be more than just a phenotype of postural BP dysregulation.

The intra-relationship between orthostatic SBP change and office-to-awake SBP differences was replicated at the second-visit measurement, despite the poor reproducibility of the orthostatic BP change. Irreproducibility of orthostatic BP change has been reported in an experimental study in frail elderly subjects ${ }^{21}$ and in a cohort study of older community residents. ${ }^{22}$ In contrast, masked HT has been reported to have fair-to-moderate reproducibility, ${ }^{23-26}$ even over a measurement period of 6 months. ${ }^{26}$ Our results also showed promising reproducibility of masked $\mathrm{HT}$, although the $\kappa$ coefficient was relatively small compared with previous reports, ${ }^{23-26}$ possibly 


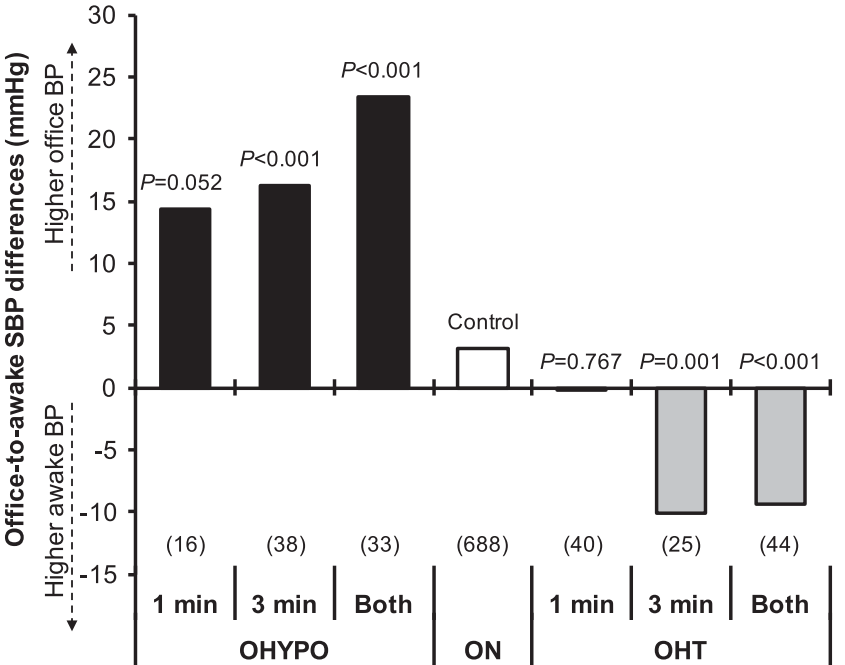

Figure 3 Effect of orthostatic BP measurement timing on office-to-awake SBP differences. Orthostatic hypertension (OHT) and hypotension (OHYPO) were defined as a maximum postural SBP change $\geqslant 10 \mathrm{~mm} \mathrm{Hg}$ and $\leqslant-20 \mathrm{~mm} \mathrm{Hg}$, respectively. OHT and OHYPO subjects were classified into three groups according to the time when the orthostatic SBP change exceeded the threshold: $1 \mathrm{~min}$, in which orthostatic SBP change exceeded the threshold only $1 \mathrm{~min}$ after standing; $3 \mathrm{~min}$, in which orthostatic SBP change exceeded the threshold only 3 min after standing; and both, in which orthostatic SBP change exceeded the threshold both 1 and 3 min after standing. One subject who showed an orthostatic SBP change of $+10 \mathrm{~mm} \mathrm{Hg}$ at $1 \mathrm{~min}$ and $-13 \mathrm{~mm} \mathrm{Hg}$ at $3 \mathrm{~min}$ was classified as OHT in this figure but was considered as having orthostatic normotension in other analyses. The number of subjects in each subgroup is shown in parentheses. Subgroup differences in the office-to-home SBP differences were assessed by analysis of variance, and Dunnett's test was used in the post-hoc analysis.

because of the long interval between the first- and second-visit measurements. These previous and present findings suggest that excessive postural BP increase, even if episodic, might imply the presence of masked HT. The higher determination coefficient obtained in the regression model that included the orthostatic BP change as an independent variable (Table 2) supports these conclusions. Although further studies with repeated measurements will be needed to corroborate our conclusions, our results will help to clarify why a one-point measurement of OHT has been associated with cardiovascular outcomes.

We did not consider antihypertensive medication status when calculating office-to-awake SBP differences or when defining masked and white-coat HT. Given the similar results between the treated and untreated subjects in the linear regression analyses for the SBP phenomenon and in the logistic regression analyses for masked HT, our present findings might be independent of antihypertensive status. A large-scale longitudinal analysis ${ }^{2}$ has previously reported that the prognostic significance of masked HT on cardiovascular outcomes is equivalent between treated and untreated subjects. In addition, when $\mathrm{BP}$ classification is made irrespective of antihypertensive drug usage, masked HT has been found to be associated with carotid atherosclerosis, ${ }^{4}$ aortic stiffness ${ }^{27}$ and renal injury. ${ }^{27}$ These results support our rationale that factors for masked HT should be investigated without distinguishing subjects by antihypertensive treatment status.

Because we compared office-to-awake BP differences with orthostatic BP change and not with BP obtained by using a simple repeated measure, we believe that our findings were relatively unaffected by

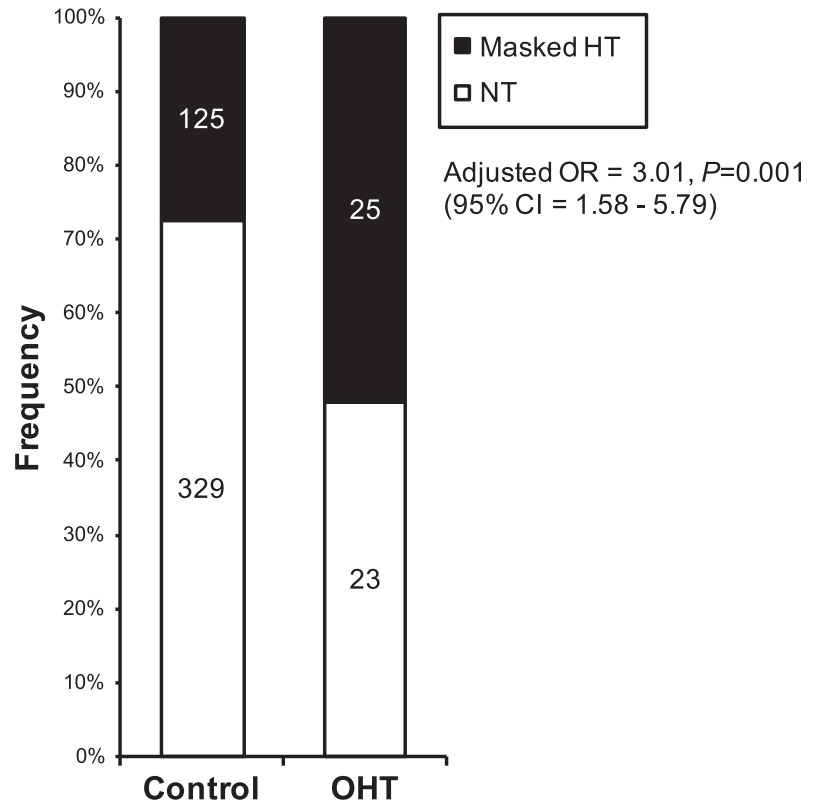

Figure 4 Frequency differences of masked hypertension by orthostatic BP status in apparently normotensive subjects $(n=502)$. Office normotensive subjects (normotension $(n=352)$ and masked HT $(n=150)$ ) were included in this analysis. Orthostatic hypertension (OHT) was defined as a postural SBP change $\geqslant 10 \mathrm{~mm} \mathrm{Hg} 3 \mathrm{~min}$ after standing. The odds ratio (OR) for masked HT was calculated using a logistic regression analysis adjusted for age, sex, body mass index and baseline office SBP.

regression to the mean, which is a statistical phenomenon caused by random error in repeatedly measured values. ${ }^{28}$ In addition, the lack of association between OHT at $1 \mathrm{~min}$ with office-to-awake SBP differences further negates the possibility that the present findings were simply a consequence of statistical error.

Several limitations to the present study are worth mentioning. First, given that we measured out-of-office BP by using ABPM, masked and white-coat HT were defined on the basis of the difference between office-measured BPs and average awake BPs, whereas BP measured by the subjects themselves in the morning (home BP) were usually used for the calculation of masked and white-coat HT. Second, ABPM was provided as an optional examination for those who requested to undergo such a procedure, which resulted in the exclusion of a large number of subjects. Third, repeated measurements were performed in only a small number of subjects who participated in the medical check-up program, which was performed of their own volition. We therefore cannot exclude the possibility of selection bias in this subpopulation. Fourth, because this study was cross-sectional in nature, we were unable to evaluate the prognostic significance of OHT and masked HT. Whether poor prognosis of OHT is just an epiphenomenon of masked HT should therefore be clarified in future studies.

In conclusion, we clarified the presence of an intra-individual relationship between postural BP change and office-to-awake BP differences, and subjects with OHT were likely to have masked HT irrespective of antihypertensive medication status. The present findings will help clarify the diagnostic and prognostic significance of BP variability, particularly orthostatic BP dysregulation.

\section{CONFLICT OF INTEREST}

The authors declare no conflict of interest. 


\section{ACKNOWLEDGEMENTS}

We thank Yoko Ochi and Hideka Fuyuki for their help in collecting the clinical data. We also thank the editors of DMC Corporation for their help in the preparation of this manuscript. This study was supported by a Grant-in-Aid for Scientific Research from The Ministry of Education, Culture, Sports, Science and Technology of Japan; The Ministry of Health, Labour and Welfare of Japan; The Japan Arteriosclerosis Prevention Fund; and a Research Promotion Award from Ehime University.

\section{THE J-SHIPP STUDY GROUP}

Chairperson: Yasuharu Tabara ${ }^{1,2}$. Research members: Katsuhiko Kohara $^{3}$, Michiya Iagase ${ }^{2}$, Tokihisa Nagai ${ }^{2,4}$, Yoko Okada ${ }^{2}$, Maya Ohara $^{2}$, Eri Uetani ${ }^{2}$, Tomoko Kido ${ }^{2}$, Namiko Ochi², Rie Takita ${ }^{2}$, Yasumasa Ohyagi ${ }^{2}$, Tetsuro Miki ${ }^{1,2}$

${ }^{1}$ Center for Genomic Medicine, Kyoto University Graduate School of Medicine, Sakyo-ku, Kyoto, Japan; ${ }^{2}$ Department of Geriatric Medicine, Ehime University Graduate School of Medicine, Toon City, Ehime, Japan; ${ }^{3}$ Department of Neurology, National Hospital Organization Ehime Medical Center, Toon City, Ehime, Japan; ${ }^{4}$ Medical Education Center, Ehime University Graduate School of Medicine, Toon City, Ehime, Japan.

1 O'Brien E, Parati G, Stergiou G, Asmar R, Beilin L, Bilo G, Clement D, de la Sierra A, de Leeuw P, Dolan E, Fagard R, Graves J, Head GA, Imai Y, Kario K, Lurbe E, Mallion JM, Mancia G, Mengden T, Myers M, Ogedegbe G, Ohkubo T, Omboni S, Palatini P, Redon J, Ruilope LM, Shennan A, Staessen JA, vanMontfrans G, Verdecchia P, Waeber B, Wang J, Zanchetti A, Zhang Y, European Society of Hypertension Working Group on Blood Pressure Monitoring. European Society of Hypertension position paper on ambulatory blood pressure monitoring. J Hypertens 2013; 31: 1731-1768.

2 Stergiou GS, Asayama K, Thijs L, Kollias A, Niiranen TJ, Hozawa A, Boggia J, Johansson JK, Ohkubo T, Tsuji I, Jula AM, Imai Y, Staessen JA, International Database on HOme blood pressure in relation to Cardiovascular Outcome (IDHOCO) Investigators. Prognosis of white-coat and masked hypertension: International Database of HOme blood pressure in relation to Cardiovascular Outcome. Hypertension 2014; 63: 675-682.

3 Cuspidi C, Sala C, Tadic M, Rescaldani M, Grassi G, Mancia G. Non-dipping pattern and subclinical cardiac damage in untreated hypertension: a systematic review and meta-analysis of echocardiographic studies. Am J Hypertens 2015; 28: 1392-1402.

4 Fukuhara M, Arima H, Ninomiya T, Hata J, Hirakawa Y, Doi Y, Yonemoto K, Mukai N Nagata M, Ikeda F, Matsumura K, Kitazono T, Kiyohara Y. White-coat and masked hypertension are associated with carotid atherosclerosis in a general population: the Hisayama study. Stroke 2013; 44: 1512-1517.

5 Franklin SS, Thijs L, Li Y, Hansen TW, Boggia J, Liu Y, Asayama K, Björklund-Bodegård $K$, Ohkubo $T$, Jeppesen J, Torp-Pedersen $C$, Dolan $E$, Kuznetsova T, Stolarz-Skrzypek K, Tikhonoff V, Malyutina S, Casiglia E, Nikitin Y, Lind L, Sandoya E, Kawecka-Jaszcz K, Filipovsky J, Imai Y, Wang J, Ibsen H, O'Brien E, Staessen JA, International Database on Ambulatory blood pressure in Relation to Cardiovascular Outcomes Investigators. Masked hypertension in diabetes mellitus: treatment implications for clinical practice. Hypertension 2013; 61: 964-967.

6 Hänninen MR, Niiranen TJ, Puukka PJ, Mattila AK, Jula AM. Determinants of masked hypertension in the general population: the Finn-Home study. J Hypertens 2011; 29: 1880-1888.

7 Eigenbrodt ML, Rose KM, Couper DJ, Arnett DK, Smith R, Jones D. Orthostatic hypotension as a risk factor for stroke: the atherosclerosis risk in communities (ARIC) study, 1987-1996. Stroke 2000; 31: 2307-2313.
8 Fagard $\mathrm{RH}$, De Cort P. Orthostatic hypotension is a more robust predictor of cardiovascular events than nighttime reverse dipping in elderly. Hypertension 2010; 56 56-61.

9 Rose KM, Tyroler HA, Nardo CJ, Arnett DK, Light KC, Rosamond W, Sharrett AR, Szklo M. Orthostatic hypotension and the incidence of coronary heart disease: the Atherosclerosis Risk in Communities study. Am J Hypertens 2000; 13: 571-578.

10 Verwoert GC, Mattace-Raso FU, Hofman A, Heeringa J, Stricker BH, Breteler MM, Witteman JC. Orthostatic hypotension and risk of cardiovascular disease in elderly people: the Rotterdam study. J Am Geriatr Soc 2008; 56: 1816-1820.

11 Fedorowski A, Stavenow L, Hedblad B, Berglund G, Nilsson PM, Melander 0. Orthostatic hypotension predicts all-cause mortality and coronary events in middleaged individuals (The Malmo Preventive Project). Eur Heart J 2010; 31: 85-91.

12 Eguchi K, Kario K, Hoshide S, Hoshide Y, Ishikawa J, Morinari M, Hashimoto T, Shimada K. Greater change of orthostatic blood pressure is related to silent cerebral infarct and cardiac overload in hypertensive subjects. Hypertens Res 2004; 27: 235-241.

13 Yatsuya H, Folsom AR, Alonso A, Gottesman RF, Rose KM, ARIC Study Investigators. Postural changes in blood pressure and incidence of ischemic stroke subtypes: the ARIC study. Hypertension 2011; 57: 167-173.

14 Kohara K, Tabara Y, Yamamoto Y, Miki T. Orthostatic hypertension: another orthostatic disorder to be aware of. J Am Geriatr Soc 2000; 48: 1538-1539.

15 Ricci F, De Caterina R, Fedorowski A. Orthostatic hypotension: epidemiology, prognosis, and treatment. J Am Coll Cardiol 2015; 66: 848-860.

16 Nardo CJ, Chambless LE, Light KC, Rosamond WD, Sharrett AR, Tell GS, Heiss G. Descriptive epidemiology of blood pressure response to change in body position. The ARIC study. Hypertension 1999; 33: 1123-1129.

17 Barochiner J, Cuffaro PE, Aparicio LS, Alfie J, Rada MA, Morales MS, Galarza CR, Waisman GD. Predictors of masked hypertension among treated hypertensive patients: an interesting association with orthostatic hypertension. Am J Hypertens 2013; 26: 872-878.

18 Tabara $\mathrm{Y}$, Okada $\mathrm{Y}$, Ohara M, Uetani E, Kido T, Ochi N, Nagai T, Igase M, Miki T, Matsuda F, Kohara K. Association of postural instability with asymptomatic cerebrovascular damage and cognitive decline: the Japan Shimanami Health Promoting Program study. Stroke 2015; 46: 16-22.

19 Tabara Y, Okada Y, Uetani E, Nagai T, Igase M, Kido T, Ochi N, Ohara M, Takita R, Kohara K, Miki T. Postprandial hypotension as a risk marker for asymptomatic lacunar infarction. J Hypertens 2014; 32: 1084-1090.

20 Winnicki M, Canali C, Mormino P, Palatini P. Ambulatory blood pressure monitoring editing criteria: is standardization needed? Hypertension and Ambulatory Recording Venetia Study (HARVEST) Group, Italy. Am J Hypertens 1997; 10: 419-427.

21 Belmin J, Abderrhamane M, Medjahed S, Sibony-Prat J, Bruhat A, Bojic N, Marquet T. Variability of blood pressure response to orthostatism and reproducibility of the diagnosis of orthostatic hypotension in elderly subjects. J Gerontol A Biol Sci Med Sci 2000; 55: M667-M671.

22 Alli C, Avanzini F, Bettelli G, Colombo F, Corso R, Di Tullio M, Marchioli R, Mariotti G, Radice M, Taioli E, Terzian E, Tognoni G, Zussino A. Prevalence and variability of orthostatic hypotension in the elderly. Results of the Italian study on blood pressure in the elderly (SPAA). Eur Heart J 1992; 13: 178-182.

23 Viera AJ, Lin FC, Tuttle LA, Olsson E, Stankevitz K, Girdler SS, Klein JL, Hinderliter AL. Reproducibility of masked hypertension among adults 30 years or older. Blood Press Monit 2014; 19: 208-215.

24 Viera AJ, Hinderliter AL, Kshirsagar AV, Fine J, Dominik R. Reproducibility of masked hypertension in adults with untreated borderline office blood pressure: comparison of ambulatory and home monitoring. Am J Hypertens 2010; 23: 1190-1197.

25 Ben-Dov IZ, Ben-Arie L, Mekler J, Bursztyn M. Reproducibility of white-coat and masked hypertension in ambulatory BP monitoring. Int J Cardiol 2007; 117: 355-359.

26 Kawabe H. Saito I. Reproducibility of masked hypertension determined from morning and evening home blood pressure measurements over a 6-month period. Hypertens Res 2007; 30: 845-851.

27 Tientcheu D, Ayers C, Das SR, McGuire DK, de Lemos JA, Khera A, Kaplan N, Victor R, Vongpatanasin W. Target organ complications and cardiovascular events associated with masked hypertension and white-coat hypertension: analysis from the Dallas Heart Study. J Am Coll Cardiol 2015; 66: 2159-2169.

28 Barnett AG, van der Pols JC, Dobson AJ. Regression to the mean: what it is and how to deal with it. Int J Epidemiol 2005; 34: 215-220.

Supplementary Information accompanies the paper on Hypertension Research website (http://www.nature.com/hr) 